\title{
A NOTE ON THE UNIQUENESS OF THE CANONICAL CONNECTION OF A NATURALLY REDUCTIVE SPACE
}

\author{
CARLOS OLMOS AND SILVIO REGGIANI
}

\begin{abstract}
We extend the result in J. Reine Angew. Math. 664, 29-53, to the non-compact case. Namely, we prove that the canonical connection on a simply connected and irreducible naturally reductive space is unique, provided the space is not a sphere, a compact Lie group with a bi-invariant metric or its symmetric dual. In particular, the canonical connection is unique for the hyperbolic space when the dimension is different from three. We also prove that the canonical connection on the sphere is unique for the symmetric presentation. Finally, we compute the full isometry group (connected component) of a compact and locally irreducible naturally reductive space.
\end{abstract}

\section{INTRODUCTION AND PRELIMINARIES}

Élie Cartan, in the 1920s, asked for linear connections, on a given Riemannian space, that adapt to the geometry in a more suitable way than the Levi-Civita connection Car24. He proposed to study the so-called connections with skewtorsion. Such connections are characterized by the property of having parallel metric tensor and the same geodesics as the Levi-Civita connection.

Spaces with skew-torsion have an increasing interest in recent years because of their applications to theoretical physics (see Agr06). A distinguished family of Riemannian spaces with skew-torsion are the naturally reductive spaces. In fact, the canonical connection $\nabla^{c}$ of a naturally reductive space $M=G / H$ provides a metric connection and has skew-torsion $T=-2\left(\nabla-\nabla^{c}\right)$, where $\nabla$ is the LeviCivita connection. If $M$ is a symmetric space, then the Levi-Civita connection is a canonical connection.

In a naturally reductive space one has that $\nabla^{c} R=0$ and $\nabla^{c} T=0$, where $R$ is the Riemannian curvature tensor. More generally, any $G$-invariant tensor on $M$ must be parallel with respect to the canonical connection.

In OR12 it was proved that the canonical connection of a (locally irreducible) compact naturally reductive space is unique, provided the space is different from the following symmetric spaces: spheres, real projective spaces and compact Lie groups with a bi-invariant metric.

The proof given in OR12 uses strongly the compactness assumption (besides the so-called skew-torsion holonomy theorem). Namely, it makes use of a decomposition theorem for compact homogeneous spaces which is false in the non-compact case

Date: September 25, 2018.

2010 Mathematics Subject Classification. Primary 53C30; Secondary 53C35.

Key words and phrases. Naturally reductive, canonical connection, skew-symmetric torsion, isometry group.

Supported by Universidad Nacional de Córdoba and CONICET. Partially supported by ANCyT, Secyt-UNC and CIEM. 
(however, such a decomposition theorem was crucial in the proof the skew-torsion holonomy theorem).

The purpose of this note is to prove that the canonical connection is unique also for simply connected (irreducible) non-compact naturally reductive spaces, with the only exceptions of dual symmetric spaces of compact Lie groups. In particular, the canonical connection is unique for any real hyperbolic space $M=H^{n}$ with $n \neq 3$ (in contrast with the compact case where many spheres are excluded).

Observe that the main result of this article, stated precisely in Theorem 2.1 also has a local version, since a canonical connection on a naturally reductive space lifts to the universal cover.

In order to prove Theorem 2.1. we use some auxiliary facts that we want to mention. Namely, that the real hyperbolic space $H^{n}$ admits a unique naturally reductive presentation (the symmetric pair presentation). This allows to prove that the canonical connection on $H^{n}$ is unique for all $n \neq 3$. To do this, we use that the canonical connection on the sphere $S^{n}$, with $n \neq 3$, is unique if we fix the symmetric presentation $S^{n}=\mathrm{SO}(n+1) / \mathrm{SO}(n)$, since from Hodge theory there are no non-trivial parallel 3-forms (see Remark 2.5)

Finally, in Section 3, we explicitly compute the isometry group of a compact and locally irreducible naturally reductive space. This extends the result in Reg10 for normal homogeneous spaces (and known results by Onishchik Oni92 and Shankar Sha01] on isometry groups of homogeneous spaces).

1.1. Skew-torsion holonomy systems. In a previous work OR12 we deal with the concept of skew-torsion holonomy systems, which are a variation of the so-called holonomy systems introduced by J. Simons in Sim62]. Skew-torsion holonomy systems arise in a natural way and in a geometric context, by considering the difference tensor between two metric connections which have the same geodesics as the Levi-Civita connection.

We say that a triple $[\mathbb{V}, \Theta, G]$ is a skew-torsion holonomy system provided $\mathbb{V}$ is an Euclidean space, $G$ is a connected Lie subgroup of $\operatorname{SO}(\mathbb{V})$, and $\Theta$ is a totally skew-symmetric 1 -form on $\mathbb{V}$ which takes values in the Lie algebra $\mathfrak{g}$ of $G$ (i.e., $(x, y, z) \mapsto\left\langle\Theta_{x} y, z\right\rangle$ is an algebraic 3 -form on $\left.\mathbb{V}\right)$. We say that $[\mathbb{V}, \Theta, G]$ is irreducible if $G$ acts irreducibly on $\mathbb{V}$, transitive if $G$ is transitive on the sphere of $\mathbb{V}$, and symmetric if $g_{*}(\Theta)=\Theta$ for all $g \in G$, where $g_{*}(\Theta)_{x}=g \circ \Theta_{g^{-1}(x)} \circ g^{-1}$.

The main result on skew-torsion holonomy systems is analogous to Simons holonomy theorem for classical holonomy systems. Such a result is actually stronger because transitive cases cannot occur others than the full orthogonal group.

Theorem 1.1 (Skew-torsion Holonomy Theorem Nag07, OR12]). Let [V, $\Theta, G]$, $\Theta \neq 0$, be an irreducible skew-torsion holonomy system with $G \neq \mathrm{SO}(\mathbb{V})$. Then $[\mathbb{V}, \Theta, G]$ is symmetric and non-transitive. Moreover,

(1) $(\mathbb{V},[\cdot, \cdot])$ is an orthogonal simple Lie algebra, of rank at least 2 , with respect to the bracket $[x, y]=\Theta_{x} y$;

(2) $G=\operatorname{Ad}(H)$, where $H$ is the connected Lie group associated to the Lie algebra $(\mathbb{V},[\cdot, \cdot])$

(3) $\Theta$ is unique, up to a scalar multiple. 


\section{The Uniqueness of the CANONicAl CONNECTiOn}

In this section we prove a uniqueness result for canonical connections on naturally reductive spaces, compact or not.

Theorem 2.1. Let $M$ be a simply connected and irreducible naturally reductive space. Assume that $M$ is not (globally) isometric to a sphere, nor to a Lie group with a bi-invariant metric or its symmetric dual. Then, the canonical connection on $M$ is unique.

Observe that, in particular, the above theorem says that the canonical connection is unique for the real hyperbolic space $H^{n}$ for all $n \neq 3$. When $n=3, H^{3}$ is the symmetric dual of $S^{3}=\mathrm{SU}(2)$, and in this case $H^{3}$ admits a line of canonical connections (see Remark 2.5).

Before giving the proof of Theorem 2.1 we fix some notation and we state some basic results we will need.

Let $M=G / G_{p}, p \in M$, be a naturally reductive space. That is, assume that $M$ carries a $G$-invariant metric and the Lie algebra of $G$ admits a decomposition $\mathfrak{g}=\mathfrak{g}_{p} \oplus \mathfrak{m}$, where $\mathfrak{g}_{p}=\operatorname{Lie}\left(G_{p}\right)$ and $\mathfrak{m}$ is an $\operatorname{Ad}\left(G_{p}\right)$-invariant subspace such that the geodesics through $p$ are given by

$$
\operatorname{Exp}(t X) \cdot p, \quad X \in \mathfrak{m} .
$$

That is to say, Riemannian geodesics coincide with $\nabla^{c}$-geodesics, where $\nabla^{c}$ is the canonical connection associated with the above mentioned reductive decomposition.

Suppose that $\nabla^{c^{\prime}}$ is another canonical connection on $M$ (associated with another naturally reductive presentation or another reductive decomposition). It follows from [OR12, Section 6] that

$$
\Theta=\left(\nabla^{c^{\prime}}-\nabla^{c}\right)_{p},
$$

that is the difference between $\nabla^{c^{\prime}}$ and $\nabla^{c}$, evaluated at $p$, is a totally skewsymmetric 1-form on $T_{p} M$ which takes values in the full isotropy subalgebra $\mathfrak{h}=$ $\operatorname{Lie}\left(\operatorname{Iso}(M)_{p}\right)$. Hence, $\left[T_{p} M, \Theta, H\right]$ is a skew-torsion holonomy system, where $H=$ $\left(\operatorname{Iso}(M)_{p}\right)^{o}$ is the connected component of the full isotropy subgroup at $p$.

Let $\tilde{\mathfrak{h}}$ be the linear span of $\left\{h_{*}(\Theta)_{v}: h \in H, v \in T_{p} M\right\}$. We have that $\tilde{\mathfrak{h}}$ is an ideal of $\mathfrak{h}$. Let $\tilde{H}$ be the connected Lie subgroup of $H$ with Lie algebra $\tilde{\mathfrak{h}}$. From OR12, Section 2] there exist decompositions

$$
T_{p} M=\mathbb{V}_{0} \oplus \mathbb{V}_{1} \oplus \cdots \oplus \mathbb{V}_{k} \quad \text { (orthogonal sum) }
$$

and

$$
\tilde{H}=H_{1} \times \cdots \times H_{k} \quad \text { (almost direct product) }
$$

such that $H_{i}$ acts trivially on $\mathbb{V}_{j}$ if $i \neq j$ (in particular, $\mathbb{V}_{0}$ is the set of fixed vectors of $\tilde{H})$ and $H_{i}$ acts irreducibly on $\mathbb{V}_{i}$ with $\mathscr{C}_{i}\left(\mathfrak{h}_{i}\right)=\{0\}$, where

$$
\mathscr{C}_{i}\left(\mathfrak{h}_{i}\right):=\left\{B \in \mathfrak{s o}\left(\mathbb{V}_{i}\right):\left[B, \mathfrak{h}_{i}\right]=0\right\} .
$$

Moreover, we have that $H$ splits as

$$
H=H_{0} \times \tilde{H}=H_{0} \times H_{1} \times \cdots \times H_{k},
$$

where $H_{0}$ acts only on $\mathbb{V}_{0}$ (and it could be arbitrary). In fact, any skew-torsion holonomy system can be decomposed in this way (see OR12] and also [AF04, Nag07). 
In order to prove Theorem 2.1, we will make use of the following basic facts.

Lemma 2.2 (see OR12]). Let $M=G / G_{p}$ be a Riemannian homogeneous manifold, let $H$ be a normal subgroup of $G_{p}$ and let $\mathbb{W}$ be the subspace of $T_{p} M$ defined by

$$
\mathbb{W}=\left\{v \in T_{p} M: d h(v)=v \text { for all } h \in H\right\} .
$$

Then $\mathbb{W}$ is $G_{p}$-invariant. Moreover, if $\mathscr{D}$ is the $G$-invariant distribution on $M$ defined by $\mathscr{D}(p)=\mathbb{W}$, then $\mathscr{D}$ is integrable with totally geodesic leaves (or, equivalently, $\mathscr{D}$ is autoparallel).

Lemma 2.3. Let $M=G / G_{p}$ be a naturally reductive space. If $X$ is a $G$-invariant field on $M$, then $X$ is a Killing field.

Proof. Let $X$ be a $G$-invariant field on $M$ and let $D=\nabla-\nabla^{c}$ be the difference tensor between the Levi-Civita connection and a canonical connection on $M$ associated with a reductive decomposition $\mathfrak{g}=\mathfrak{g}_{p} \oplus \mathfrak{m}$. Since $X$ is $G$-invariant, then $X$ is $\nabla^{c}$-parallel (since $\nabla^{c}$ is $G$-invariant and the $\nabla^{c}$-parallel transport along the geodesic $\operatorname{Exp}(t Z) \cdot p, Z \in \mathfrak{m}$, is given by $\left.\operatorname{Exp}(t Z)_{*}\right)$. So, $\nabla X=D X$ is skew-symmetric and this implies that $X$ is a Killing field.

Proof of Theorem 2.1. We keep the notation from the previous paragraphs. We have decompositions $T_{p} M=\mathbb{V}_{0} \oplus \mathbb{V}_{1} \oplus \cdots \oplus \mathbb{V}_{k}$ (orthogonal) as in 2.1 and $H=$ $H_{0} \times \tilde{H}=H_{0} \times H_{1} \times \cdots \times H_{k}$ as in 2.2. Let $\mathbb{W}_{0}$ be the set of fixed vectors of $H$ in $T_{p} M$, via the isotropy representation. So, $\mathbb{W}_{0} \oplus \mathbb{V}_{1}$ is the set of fixed vectors of $H^{1}=H_{0} \times H_{2} \times \cdots \times H_{k}$ and hence, by Lemma 2.2, it induces the $G$-invariant autoparallel distribution $\mathscr{D}^{1}$ defined by $\mathscr{D}^{1}(p)=\mathbb{W}_{0} \oplus \mathbb{V}_{1}$.

Let $\mathscr{D}_{0}$ the $G$-invariant autoparallel distribution defined by $\mathscr{D}_{0}(p)=\mathbb{W}_{0}$. The key factor in the proof is to show that $\mathscr{D}_{0}$ is parallel along $\mathscr{D}^{1}$, and then make use of the skew-torsion holonomy theorem. Since $\mathscr{D}_{0}$ is $G$-invariant we only have to prove that $\mathscr{D}_{0}$ is parallel at $p\left(\right.$ along $\left.\mathscr{D}^{1}\right)$.

Let $S^{1}(p)$ be the maximal connected integral manifold of $\mathscr{D}^{1}$ which contains $p$. That is, $S^{1}(p)$ is the set of fixed points of $H^{1}$ on $M$ (connected component). It is not difficult to see that $S^{1}(p)$ is an extrinsic homogeneous submanifold under the action of the group

$$
G^{1}(p)=\left\{g \in G: g\left(S^{1}(p)\right)=S^{1}(p)\right\}=\left\{g \in G: g(p) \in S^{1}(p)\right\}
$$

with effective isotropy $H_{1}$. Recall that the metric on $S^{1}(p)$ is naturally reductive, since $S^{1}(p)$ is a totally geodesic submanifold of $M$.

Let $X \in \mathbb{W}_{0}$ and let $\tilde{X}$ be the $G^{1}(p)$-invariant field on $S^{1}(p)$ such that $\tilde{X}(p)=X$, or equivalently, the restriction to $S^{1}(p)$ of the $G$-invariant field on $M$ with initial condition $X$. It follows from Lemma 2.3 that $\tilde{X}$ is a Killing field and hence, its derivative $\nabla \tilde{X}$ is skew-symmetric.

Observe that if $h \in H_{1}$ and $v \in \mathbb{W}_{0} \oplus \mathbb{V}_{1} \simeq T_{p} S^{1}(p)$, then

$$
d h\left(\nabla_{v} \tilde{X}\right)=\nabla_{d h(v)} h_{*}(\tilde{X})=\nabla_{d h(v)} \tilde{X} .
$$

Then $(\nabla \tilde{X})_{p}$ commutes with $H_{1}$ (via the isotropy representation) and so, it leaves $\mathbb{W}_{0}$ and $\mathbb{V}_{1}$ invariant. Since $\mathscr{C}_{1}\left(\mathfrak{h}_{1}\right)=\{0\}$ we have that $\left.(\nabla \tilde{X})_{p}\right|_{\mathbb{V}_{1}} \equiv 0$, and therefore $\nabla_{v} \tilde{X} \in \mathbb{W}_{0}$ for all $v \in \mathbb{W}_{0} \oplus \mathbb{V}_{1}$. This implies that $\mathscr{D}_{0}$ is parallel along $\mathscr{D}^{1}$. Then, $\mathscr{D}_{1}$ is parallel along $\mathscr{D}^{1}$ and hence, $\mathscr{D}_{1}$ is autoparallel on $M$, since $\mathscr{D}^{1}$ is autoparallel. On the other side, we have that $\mathscr{D}_{1}^{\perp}$ is also an autoparallel distribution on $M$, since 
$\mathscr{D}_{1}^{\perp}(p)=\mathbb{V}_{0} \oplus \mathbb{V}_{2} \oplus \cdots \oplus \mathbb{V}_{k}$ is the set of fixed vectors of $H_{1}$, and therefore $M$ splits off, unless these distributions are trivial.

Finally, we reach two possibilities:

(1) $T_{p} M=\mathbb{V}_{0}$ and $H=\left(\operatorname{Iso}(M)_{p}\right)^{o}=H_{0}$, or

(2) $T_{p} M=\mathbb{V}_{1}$ and $H=\left(\operatorname{Iso}(M)_{p}\right)^{o}=H_{1}$.

In the first case, we have that the group $\tilde{H}$ spanned by $\nabla^{c^{\prime}}-\nabla^{c}$ is trivial, and then we conclude that $\nabla^{c}=\nabla^{c^{\prime}}$.

In the second case, we have two possibilities again. Firstly, if $H_{1}$ is transitive on the unit sphere of $T_{p} M$, then, by using the skew-torsion holonomy theorem, we have that $H_{1}=\left(\operatorname{Iso}(M)_{p}\right)^{o}=\mathrm{SO}\left(T_{p} M\right)$. So, it is standard to see that $M=S^{n}$ or $M=H^{n}$. See Proposition 2.4 and Remark 2.5 below to exclude the hyperbolic case when $n \neq 3$. On the other hand, if $H_{1}$ is not transitive on the sphere, the skewtorsion holonomy theorem says that $H_{1}$ acts on $T_{p} M$ as the adjoint representation of a simple and compact Lie group. If $M$ is compact, it follows from the classification of strongly isotropy irreducible spaces, given by J. Wolf in [Wol68] (see OR12, Appendix] for a conceptual proof), that $M$ is a Lie group with a bi-invariant metric. If $M$ is non-compact, then $M$ turns out a symmetric space (since non-compact isotropy irreducible spaces must be symmetric [Bes87, WZ91]). If $\nabla^{c} \neq \nabla^{c^{\prime}}$, we have, by taking the symmetric dual, that $M^{*}$ is isometric to a Lie group with a bi-invariant metric. In fact, it is not difficult to see that there is a one-one correspondence between canonical connections on $M$ and canonical connections on $M^{*}$ (see Remark 2.6).

This completes the proof of Theorem 2.1.

Proposition 2.4. The real hyperbolic space $H^{n}$ admits a unique naturally reductive presentation, the symmetric pair decomposition $H^{n}=\mathrm{SO}(n+1,1)^{\circ} / \mathrm{SO}(n)$.

Proof. Let $G$ be a connected Lie subgroup of $\operatorname{Iso}\left(H^{n}\right)$ which acts transitively on $H^{n}$ and such that $H^{n}=G / H$ is a naturally reductive space. If $G$ is semisimple, it is standard to show that $G=\operatorname{Iso}\left(H^{n}\right)^{o}=\mathrm{SO}(n+1,1)^{o}$. In fact, let $K$ be a maximal compact subgroup of $G$. So, $K$ has a fixed point, say $p$. We may assume that $H$ is the isotropy group at $p$. So $H=K$, since $K$ is maximal. Hence, $(G, H)$ is presentation of $H^{n}$ as an effective Riemannian symmetric pair, and therefore $G=\mathrm{SO}(n+1,1)^{\circ}$ (otherwise, $H^{n}$ would have two different presentations as an effective Riemannian symmetric pair).

If $G$ is not semisimple, then $G$ has a nontrivial normal abelian Lie subgroup $A$. It is a well-known fact that, either $A$ fixes a unique point at infinity or $A$ translates a unique geodesic. If $A$ translates a unique geodesic $\gamma(t)$, then $G$ leaves $\gamma$ invariant, since $A$ is a normal subgroup of $G$, and so $G$ cannot be transitive, which is a contradiction. So, let $q_{\infty}$ be the unique point at infinity which is fixed by $A$, and let $\mathscr{F}$ be the foliation on $H^{n}$ by parallel horospheres centered at $q_{\infty}$. So, we have that $A$ leaves $\mathscr{F}$ invariant, and hence $G$ does. Let $p \in H^{n}$ and let $\mathscr{F}_{p}$ be the horosphere through $p$. Denote by $\tilde{G}$ the connected component of the subgroup of $G$ which leaves $\mathscr{F}_{p}$ invariant. Then $\tilde{G}$ is transitive on $\mathscr{F}_{p}$. Hence, since $H^{n}$ is naturally reductive with respect to the decomposition $G / H$, each horosphere must be totally geodesic, a contradiction.

Remark 2.5. Let us consider the sphere $S^{n}=\mathrm{SO}(n+1) / \mathrm{SO}(n)$. Then, for all $n \neq 3$, the Levi-Civita connection is the unique canonical connection on $S^{n}$ associated 
with this naturally reductive decomposition. In fact, if $\nabla^{c}$ is another canonical connection on $S^{n}$ then, the difference tensor $D=\nabla-\nabla^{c}$ induces a $\mathrm{SO}(n+1)$ invariant 3-form $\omega(x, y, z)=\left\langle D_{x} y, z\right\rangle$. Since $\omega$ is invariant, $\omega$ is a harmonic 3-form on $S^{n}$. From Hodge theory, $\omega$ represents a nontrivial cohomology class of order 3 of the sphere $S^{n}$. This yields a contradiction, unless $n=3$.

As a consequence, it follows from Proposition 2.4 and the next remark that the real hyperbolic space $H^{n}$ admits a unique canonical connection for all $n \neq 3$. If $n=3, H^{3}$ is the dual symmetric space of the compact Lie group $S^{3} \simeq \mathrm{SU}(2)$, and therefore it admits exactly a line of canonical connections (see [OR12, Remark 6.1]).

Remark 2.6. Let $M=G / K$ be a symmetric space with associated Cartan decomposition $\mathfrak{g}=\mathfrak{k} \oplus \mathfrak{p}$. Then, there is a one-one correspondence between canonical connections on $M$ and canonical connections on the dual $M^{*}=G^{*} / K$. In fact, assume that $M$ admits a canonical connection $\nabla^{c}$ associated with a reductive decomposition $\mathfrak{g}=\mathfrak{k} \oplus \mathfrak{m}$. Let $\mathfrak{g}^{*}=\mathfrak{k} \oplus i \mathfrak{p}$ be the Lie algebra of $G^{*}$, regarded as a subspace of the complexification $\mathfrak{g}^{\mathbb{C}}$ of $\mathfrak{g}$. It is clear that $\mathfrak{m}^{*}$ (the subspace of $\mathfrak{g}^{*}$ induced by $\mathfrak{m}$, via the vector spaces isomorphism $\left.\mathfrak{g}^{*} \simeq \mathfrak{g}\right)$ is and $\operatorname{Ad}^{*}(K)$-invariant subspace such that the geodesics through $p=e K$ are given by 1-parameter subgroups with initial velocities in $\mathfrak{m}^{*}$. So, $\nabla^{c}$ corresponds to a unique canonical connection on $M^{*}$.

\section{The ISOMETRY Group of COMPACT NATURALLY REDUCTIVE SPACES}

Let $M=G / H$ be a compact and locally irreducible naturally reductive space and let $\nabla^{c}$ be the canonical connection associated with the reductive decomposition $\mathfrak{g}=$ $\mathfrak{h} \oplus \mathfrak{m}$. Assume that $M \neq S^{n}, M \neq \mathbb{R} P^{n}$. Then, from [OR12, Theorem 1.1] we have that $\operatorname{Iso}(M)^{o}=\operatorname{Aff}\left(M, \nabla^{c}\right)^{o}$, where $\operatorname{Aff}\left(M, \nabla^{c}\right)^{o}$ is the connected component of the affine group of $\nabla^{c}$ (i.e., the subgroup of diffeomorphisms of $M$ which preserve $\nabla^{c}$ ).

By making use of Lemma 2.3 and some arguments in Reg10 one can obtain the connected component of the isometry group of $M$. Actually, it is possible to simplify such arguments.

In fact, let $\operatorname{Tr}\left(M, \nabla^{c}\right)$ be the group of transvections of $\nabla^{c}$, that is, the connected Lie subgroup of $\operatorname{Aff}\left(M, \nabla^{c}\right)^{o}$ with Lie algebra $\mathfrak{t r}\left(M, \nabla^{c}\right)=[\mathfrak{m}, \mathfrak{m}]+\mathfrak{m}$ (not a direct sum, in general). Recall that $\operatorname{Tr}\left(M, \nabla^{c}\right)$ is a normal subgroup of $\operatorname{Aff}\left(M, \nabla^{c}\right)^{o}$. As it is done in Reg10 for normal homogeneous spaces, we have that $G=\operatorname{Tr}\left(M, \nabla^{c}\right)$ and thus, $G$ is a normal subgroup of $\operatorname{Aff}\left(M, \nabla^{c}\right)^{o}$. (In fact, $\mathfrak{t r}\left(M, \nabla^{c}\right)$ is an ideal of $\mathfrak{g}$, then if $\mathfrak{t r}\left(M, \nabla^{c}\right) \neq \mathfrak{g}$, since $M$ is compact, one can take a complementary ideal of the transvection algebra in $\mathfrak{g}$, which must be contained in the isotropy algebra. This is a contradiction, since we assume that $G$ acts effectively on $M$.)

Now, since $G$ is a normal subgroup of $\operatorname{Iso}(M)^{o}=\operatorname{Aff}\left(M, \nabla^{c}\right)^{o}$, we can write

$$
\mathfrak{i s o}(M)=\mathfrak{g} \oplus \mathfrak{b},
$$

where $\mathfrak{b}$ is a complementary ideal of $\mathfrak{g}$ in $\mathfrak{i s o}(M)$ (recall that $M$ is compact, and hence $\operatorname{Iso}(M)^{o}$ is also compact). Note that elements of $\mathfrak{b}$ correspond to $G$-invariant fields on $M$, which are Killing fields by Lemma 2.3 (but not any $G$-invariant field belongs to $\mathfrak{b}$, in principle).

We can summarize this fact as follows.

Theorem 3.1. Let $M=G / H$ be a compact naturally reductive space. Assume that $M$ is locally irreducible and that $M$ is not (globally) isometric to the sphere $S^{n}$ nor 
to the real projective space $\mathbb{R} P^{n}$. Then the connected component of the isometry group of $M$ is given by

$$
\operatorname{Iso}(M)^{o}=G_{\mathrm{ss}} \times K \quad \text { (almost direct product), }
$$

where $G_{\mathrm{ss}}$ is the semisimple part of $G$ and $K$ is the connected subgroup of $\operatorname{Iso}(M)$ whose Lie algebra consists of the $G$-invariant fields. In particular, Iso $(M)$ is semisimple if and only if $K$ is semisimple.

Remark 3.2. In the notation of Theorem 3.1, $K$ can be identified with (the connected component of) the set of fixed points of the isotropy group $H$, acting simply and transitively by right multiplication. Moreover, just by coping the argument in Reg10, Theorem 1.4] we get that the set of fixed points of the full isotropy group $\left(\operatorname{Iso}(M)^{o}\right)_{p}$ is a torus.

\section{REFERENCES}

[AF04] I. Agricola and Th. Friedrich, On the holonomy of connections with skew-symmetric torsion, Math. Ann. 328 (2004), no. 4, 711-748.

[Agr06] I. Agricola, The Srni lectures on non-integrable geometries with torsion, Arch. Math. (Brno) 42 (2006), no. 5, 5-84.

[Bes87] A. Besse, Einstein manifolds, Ergebnisse der Mathematik und ihrer Grenzgebiete. 3. Folge, Bd. 10, Springer-Verlag, 1987.

[Car24] É. Cartan, Les récentes généralisations de la notion d'espace, Bull. Sci. Math. 48 (1924), 294-320.

[Nag07] P.-A. Nagy, Skew-symmetric prolongations of Lie algebras and applications, arXiv:0712.1398 2 [math.DG] (2007), to appear in J. Lie Theory.

[Oni92] A. L. Onishchik, The group of isometries of a compact Riemannian homogeneous space, Differential geometry and its applications (Eger, 1989), Colloq. Math. Soc. János Bolyai, vol. 56, North-Holland, Amsterdam, 1992, pp. 597-616.

[OR12] C. Olmos and S. Reggiani, The skew-torsion holonomy theorem and naturally reductive spaces, J. Reine Angew. Math. 664 (2012), 29-53.

[Reg10] S. Reggiani, On the affine group of a normal homogeneous manifold, Ann. Global Anal. Geom. 37 (2010), no. 4, 351-359.

[Sha01] K. Shankar, Isometry groups of homogeneous spaces with positive sectional curvature, Differential Geom. Appl. 14 (2001), no. 1, 57-78.

[Sim62] J. Simons, On the transitivity of holonomy systems, Ann. of Math. 76 (1962), 213-234.

[Wol68] J. Wolf, On the geometry and structure of isotropy irreducible homogeneous spaces, Acta Math. 120 (1968), 59-148, correction: Acta Math. 152 (1984), 141-142.

[WZ91] M. Wang and W. Ziller, On isotropy irreducible Riemannian manifolds, Acta Math. 166 (1991), 223-261.

Facultad de Matemática, Astronomía y Física, Universidad Nacional de Córdoba, Ciudad Universitaria, 5000 Córdoba, Argentina

E-mail address: olmos@famaf.unc.edu.ar reggiani@famaf.unc.edu.ar 